\title{
Estrategias de docentes en centros educativos que se transforman a favor de la equidad
}

\author{
Teachers' strategies in schools that are changing towards equity
}

María García-Cano Torrico
Universidad de Córdoba
Esther Márquez Lepe
Universidad de Sevilla

Recibido: 18/09/2019

Aceptado: 27/11/2019

\begin{abstract}
This paper analyses the strategies developed by teachers working in schools that are pursuing school change projects geared towards equity and social justice. These teaching projects have emerged in schools with a view to improving school life, reducing absenteeism and academic failure among pupils. The purpose of this paper is to identify the strategies used by teachers to project pedagogical change and interpret the certainties and uncertainties generated by this change. The research is exploratory and qualitative and was conducted in two Early Years and Primary Schools selected intentionally, located in two towns in Andalusia (Spain) and in contexts of socio-economic inequality. Data were generated through semi-structured interviews conducted with teachers and the school management teams over the course of four months in each school. The findings highlight school change strategies such as: shared work, team training, teachers' personal commitment, and dialogue with families and social organisations. Differences are observed between the two contexts, which are used to interpret the certainties and uncertainties generated throughout the entire development of the school change project, and which are linked to two elements: the different stages of each of the teaching projects (in transition or consolidated) and the different ownership structures of the schools (state-owned or private/grant-maintained).
\end{abstract}

KEY WORDS: Organizational change, Primary school teachers, social justice, cultural diversity, social inequality, disadvantaged schools, primary schools, interviews

\section{RESUMEN}

En este trabajo se analizan las estrategias desarrolladas por el profesorado en centros educativos con proyectos de cambio escolar orientados a la equidad y justicia social. Estos proyectos pedagógicos surgen en escuelas con las finalidades de mejorar la convivencia, reducir el absentismo y el fracaso escolar del alumnado. El objetivo del trabajo es identificar las estrategias en las que el profesorado proyecta el cambio pedagógico e interpretar las certezas e incertidumbres que se generan ante el mismo. La investigación es de naturaleza exploratoria y cualitativa y se ha realizado en dos escuelas de educación Infantil y Primaria seleccionadas de forma intencional, localizadas en dos localidades de Andalucía (España) y en contextos de desigualdad socio-económica. Los datos se han producido a través de entrevistas semi-estructuradas realizadas al profesorado y al equipo directivo durante cuatro meses en cada centro. Los resultados destacan estrategias de cambio escolar como: el trabajo compartido, la formación en equipo, el compromiso personal del profesorado y el diálogo con familias y organizaciones sociales. Por su parte, encontramos diferencias entre ambos contextos que sirven para interpretar las certezas e incertidumbres generadas durante todo el proceso de desarrollo del proyecto de cambio escolar vinculados a dos elementos: los distintos momentos en los que se encuentra cada uno de los proyectos pedagógicos (en transición o consolidado) y la diferente titularidad de las escuelas (pública o privada-concertada).

PALABRAS CLAVE: cambio organizacional, docente, justicia social, diversidad cultural, desigualdad social, escuela desfavorecida, escuela primaria, entrevista

Autor de correspondencia:

María García-Cano Torrico, Departamento de Educación, Área de Didáctica y Organización Escolar, Universidad de Córdoba (España). E-mail: maria.garciacano@uco.es ORCID: http://orcid.org/0000-0002-0988-7280 


\section{Introducción}

Los planteamientos de la enseñanza para la equidad y la justicia social están teniendo desde hace unas décadas una mayor acogida en el debate educativo, sobre todo por los efectos que las políticas educativas neoliberales están teniendo para quienes están estigmatizados por su pertenencia ética o diferencias culturales, población pobre y migrante (Aguado-Odina, Mata-Benito y Gil-Jaurena, 2017). Desde estos espacios se propone una reformulación de prioridades y nuevas acciones políticas que cuestionen la interpretación que se hace del fracaso en las trayectorias escolares de este alumnado y sus resultados académicos.

Es precisamente desde este debate donde se somete a análisis la figura del profesorado. La literatura apunta que es uno de los ejes fundamentales en los que se apoyan los procesos de cambio escolar y sobre el que pivotan tanto las posibilidades de acción como la orientación que pueden tomar dichos procesos (Philpott y Oates, 2017). Su papel es aún más relevante en centros educativos con alta presencia de colectivos culturalmente diversos y en situaciones de desigualdad social (Wrigley, 2011; Silas-Casillas y Perales-Franco, 2014) que buscan con el cambio no sólo una mejora del rendimiento escolar sino reducir el absentismo, mejorar la convivencia y favorecer las relaciones con la comunidad y las familias. Es decir, centros que buscan superar la exclusión social, mejorar la equidad y justicia social (Lupton, 2005; Gilad-Hai y Somech, 2016). Entendemos por tanto que justicia social y equidad van más allá de la igualdad (Ayers, Quinn y Stovall, 2009), pues supone garantizar el acceso de todo el alumnado a los mismos recursos, garantizándolos sobre todo para el que está en posiciones más desfavorecidas.

Sleeter, Montecinos y Jiménez (2016) señalan cuatro dimensiones del trabajo de los docentes y las docentes en las escuelas que buscan equidad y justicia social, estas son: a) situar a las familias y a las comunidades dentro de un análisis de las desigualdades estructurales; b) desarrollar relaciones de reciprocidad con los estudiantes, las familias y las comunidades; c) enseñar teniendo altas expectativas académicas en los estudiantes, capitalizando en su cultura, lengua, experiencia e identidad, y d) elaborar y enseñar un currículo que integre las perspectivas marginadas y enfrente de manera explícita los temas de equidad y poder. En este trabajo han emergido de forma más clara las dos primeras dimensiones y atendiendo al objetivo de este trabajo se analizan a través de dos ámbitos que señala Essomba (2006) como: (1) intra, referidas a las interacciones entre el profesorado como equipo de trabajo que organiza y piensa la enseñanza en un contexto particular; e (2) inter, referidas a las estrategias emprendidas con las familias y el entorno en los que los docentes llevan a cabo su actividad.

De forma concreta, las investigaciones relativas a las estrategias de relación dentro (intra) del equipo de profesorado destacan la personalidad, el compromiso y la ética (Van der Heijden, et al., 2018) como factores principales para entender tanto las condiciones del cambio educativo en la escuela como la creación de culturas escolares de colaboración y liderazgo distribuido que lo posibiliten. Junto a ello, se destacan los principios de solidaridad, confianza y compromiso para entender el trabajo entre docentes (Wrigle et al., 2012; Moliner, Sales y Escobedo, 2016; Philpott y Oates, 2017).

Por otro lado, las investigaciones sobre escuela, familias y comunidad (inter) señalan como elemento fundamental en estos centros que buscan mayor equidad, el desarrollo de proyectos donde el currículum se diseñe para conectar con la vida y experiencias familiares de los estudiantes, estableciendo relaciones desde un ethos escolar que permite activar más voces (Aguado-Odina et al., 2017). Algunas experiencias en este sentido han sido el programa de "fondos de conocimiento" en EE.UU. o Australia (González, Moll y Amanti, 2005; Subero, Vila y Esteban-Guitart, 2015), que intentan superar el abismo existente entre el mundo de la escuela y la realidad vivida por los estudiantes, superando la perspectiva del déficit. O los trabajos realizados por Hajisoteriou y Angelides (2017) en escuelas de contextos marginados de Chipre donde destaca el trabajo del profesorado en la construcción de diálogos con las comunidades (Hajisoteriou, Karousiou y Panayiotis, 2017). En España, el modelo educativo Comunidades de Aprendizaje (CdA) está 
recibiendo especial interés en la literatura, pues la transformación no solo opera dentro de la escuela, sino con el entorno socio-cultural a través de procesos dialógicos que involucran a las familias y las comunidades dentro del centro escolar (García, Mircea y Duque, 2010; Flecha y Soler, 2013).

Para avanzar en el conocimiento de estos proyectos pedagógicos que se movilizan e imaginan la equidad y la justicia social, se muestra fundamental entender las experiencias del profesorado que participa de estos procesos de cambio, así como explorar sus estrategias reconociendo las certezas e incertidumbres que potencian o debilitan su éxito.

\section{Metodología}

El objetivo de esta investigación es identificar las estrategias en las que el profesorado proyecta el cambio escolar orientado a la equidad y justicia social. Para ello, se analizan sus discursos, sus experiencias y los elementos que median sobre sus certezas e incertidumbres.

La investigación de corte exploratoria y cualitativa presenta los resultados de un estudio de caso a través de un análisis comparado de dos escuelas de Infantil y Primaria. Esta estrategia permitió, por un lado, identificar regularidades en los discursos del profesorado, al atenderse a todos los elementos como un todo sistémico (Creswell, 2007) y, por otro, una mejor compresión de cada una de las escuelas al desvelarse la similitud/disimilitud de los discursos de los docentes en cada contexto.

Con el objeto de dotar de validez a la investigación se explicitan entre otros aspectos los criterios de selección de las escuelas, cuyo carácter fue intencional siguiendo tres criterios fundamentales. En primer lugar, para abordar la temática de nuestro estudio, es decir, procesos de cambio vinculados a la equidad y la justicia social, se seleccionaron centros que manifestaban querer mejorar el bajo rendimiento, los conflictos de convivencia y reducir las altas tasas de absentismo. En segundo lugar, para que pudiera haber comparabilidad, necesitábamos un marco normativo común en materia educativa, de forma que se eligieron escuelas situadas en una misma Comunidad Autónoma. Y, en tercer lugar, para ahondar en el conocimiento de estrategias comunes, seleccionamos contextos con características socio-demográficas similares, resultando ser escuelas ubicadas en zonas con fuerte desventaja social, alto porcentaje de alumnado extranjero y alumnado nacional de etnia gitana. (ver Tabla 1).

Tabla 1

Criterios de selección de las escuelas: $A$ y $B$

\begin{tabular}{lll}
\hline Criterios de selección & Similitud & Disimilitud \\
\hline Titularidad /ubicación & $\begin{array}{l}\text { Centros de Infantil y Primaria en } \\
\text { Andalucía (España) }\end{array}$ & $\begin{array}{l}\text { Escuela A, Pública. Población de más de } \\
500.000 \text { habitantes. }\end{array}$ \\
& & $\begin{array}{l}\text { Escuela B, Concertada. Población de entre } \\
50.000-100.000 \text { habitantes. }\end{array}$ \\
\hline Desigualdad & $\begin{array}{l}\text { Contexto: Zonas de Necesidades } \\
\text { de Transformación Social }\end{array}$ & $\begin{array}{l}\text { Escuela A: 25-45\% alumnado extranjero o de } \\
\text { etnia gitana nacional. }\end{array}$ \\
& $\begin{array}{l}\text { (ZNTS). } \\
\text { Índice Socioeconómico y Cultural } \\
\text { (ISC): "Bajo"1 }\end{array}$ & $\begin{array}{l}\text { Escuela B: 68\% de etnia gitana nacional y 25\% } \\
\text { alumnado extranjero. }\end{array}$ \\
\hline Transformación & $\begin{array}{l}\text { Escuela A: proyecto de cambio y mejora } \\
\text { consolidado. Modelo decidido por el equipo } \\
\text { de profesorado, sin protocolizar. }\end{array}$ \\
& $\begin{array}{l}\text { Cambio educativo planeado con } \\
\text { continuidad y participación de }\end{array}$ & $\begin{array}{l}\text { Escuela B: proyecto de cambio y mejora en } \\
\text { estado de transición. Modelo protocolizado en } \\
\text { equipo. }\end{array}$ \\
& $\begin{array}{l}\text { el proyecto Comunidad de Aprendizaje (en } \\
\text { adelante CdA) (Flecha y Soler, 2013). }\end{array}$ \\
\hline
\end{tabular}

Los elementos diferenciales que emergieron y se revelaron significativos para la interpretación de cada caso fueron: (1) el proyecto concreto emprendido, ya que utilizaban distintas estrategias y 
denominación; (2) la etapa en la que se sitúa cada uno de los centros, uno en transición y otro consolidada; (3), su diferente titularidad: uno público y otro concertado. Estos elementos no fueron tomados como variables observables a priori sino que, cada caso, se consideró como "unidad exploratoria" particular (Ragin, 1987). No obstante, sí se revelaron como elementos significativos no determinantes- para contextualizar e interpretar de forma significativa los discursos producidos en cada contexto.

\subsection{Contextos de estudio}

Escuela A. Colegio público de Infantil y Primaria localizado en el centro de capital de provincia y descrito por los docentes y las docentes como un barrio con población envejecida, de estrato obrero, altas tasas de desempleo y economía sumergida, toxicomanías e importantes índices de pobreza y marginación social. El barrio es catalogado como Zona con Necesidades de Transformación Social (ZNTS) en los Planes Integrales de urbanismo de la ciudad, por lo que cuenta desde los '90 con distintas actuaciones contra la exclusión social por parte de las administraciones. La presencia de población inmigrante extranjera en el barrio se estima el triple que en resto de la ciudad ( $40 \%$ en el barrio frente al 8\% de la ciudad según el Padrón Municipal, 2017), la población nacional de etnia gitana es el colectivo mayoritario.

El proyecto pedagógico en el colegio se inició en el año 2002 impulsado por 8 maestros y maestras en un proceso de diálogo entre el equipo docente y la administración educativa, lo que supuso un cambio con lo que se venía realizando anteriormente. En la actualidad los pilares de trabajo son: (1) la identidad y autoestima del alumnado, (2) mejora de la convivencia, (3) relaciones con el entorno y las familias y (4) desarrollo del currículum. Para ello, las principales estrategias son: las asambleas, la presencia de dos maestras o maestros por aula, agrupamientos flexibles de espacios, tiempos y encuentros inter-niveles entre el alumnado o la creatividad como herramienta educativa. El proyecto se considera consolidado por el equipo después de 15 años de desarrollo, aunque sometido a diálogo y constantes revisiones.

La Escuela B de Infantil y Primaria es de titularidad concertada, religiosa-católica. Está dirigido por una Congregación con una red de centros localizados por todo el país, con un mismo ideario y organización de tipo piramidal, donde la dirección decide los proyectos en los que se implica cada una de sus escuelas. Se localiza en una ciudad de tamaño medio, en un barrio de zona periférica considerada tradicionalmente marginal, tal como describe el propio equipo de profesorado natural de la población. Declarada también ZNTS debido al: deterioro urbanístico y déficit en infraestructura, altos índices de absentismo y fracaso escolar, altas tasas de desempleo y deficiencias higiénicosanitarias. En la ciudad, según el profesorado, se reconoce como zona de tráfico de armas, drogas y su consumo. Según los últimos datos del Padrón Municipal del 2016 el porcentaje de población extranjera del distritito es doce veces el de la ciudad (25\% del barrio frente al 2,31\% de la ciudad). En este contexto, se describe un clima de desmotivación por parte del profesorado que fue canalizado por la dirección con la propuesta de desarrollo del proyecto Comunidades de Aprendizaje (Flecha y Soler, 2013), iniciado en el curso 2009/10 con el acuerdo de toda la plantilla. Sus principales objetivos fueron la apertura de la escuela a la comunidad y ofrecer una mayor calidad en la educación del alumnado y sus familias. En la actualidad, el proyecto de transformación sigue un esquema planificado que implica estrategias definidas desde fuera entre las que se encuentran: definición de metas compartidas (el sueño), creación de grupos interactivos dentro del aula, presencia de adultos en las aulas, celebración de tertulias literarias, creación de comisiones mixtas (profesorado, alumnado y otros agentes) y formación de familiares. El equipo docente considera el proyecto aún en una fase intermedia.

\subsection{Instrumentos y análisis}

El principal instrumento de investigación ha sido la entrevista semiestructurada complementada con observaciones informales en distintos espacios (aula, reuniones de profesorado y encuentros con familias), ya que el trabajo de campo se desarrolló durante cuatro meses en cada 
uno de los centros. Se realizaron un total de 25 entrevistas al profesorado de los dos colegios: 5 a miembros del equipo directivo ( 3 en el centro A y 2 en el centro B) y 20 a profesorado de diferentes cursos de una duración de entre una hora y media y dos horas (9 en el centro A y 11 en el centro B). Para realizarlas se obtuvieron los permisos necesarios de la dirección de los colegios y se acordó la devolución de los resultados atendiendo a sus necesidades expresadas. El número de entrevistas vino determinado por el principio de saturación.

El guion se estructuró sobre los 11 aspectos detectados por Sammons (1999), adaptados y examinados por Lupton (2005) para escuelas con proyectos hacia la justicia social: liderazgo profesional, visión y objetivos compartidos, ambiente de aprendizaje, concentración en la enseñanza y el aprendizaje, enseñanza útil, expectativas, refuerzo positivo, seguimiento del progreso, derechos y responsabilidades del alumnado, asociación de hogar / escuela, organización del aprendizaje.

La transcripción literal de las entrevistas se codificó en el soporte informático Atlas.ti v7.1. prioritariamente de forma selectiva: por un lado, con códigos que respondían a las dimensiones del guion de entrevista según la propuesta de Lupton (2005); por otro lado, los resultados se organizaron en las dos dimensiones señaladas en la introducción que explicita Essomba (2006): intra e inter. Se sigue este esquema en la presentación de los resultados y se toman como ejes comparativos para favorecer su interpretación: la diferente titularidad de los colegios (público y privado-concertado) y el tiempo de desarrollo del proyecto (en transición y consolidado).

\section{Resultados}

3.1. La dimensión intra: profesorado eje del cambio

Los diferentes momentos por los que transcurren los proyectos de cambio se han mostrado muy significativos para interpretar el discurso del profesorado, especialmente en relación a sus certezas e incertidumbres, ya que encontramos regularidades en el discurso del profesorado según se refieran a los inicios, la acomodación o consolidación del proyecto.

Encontramos que en la mayor parte de los discursos analizados, cuando el profesorado narra la primera toma de contacto con el proyecto de cambio escolar, reconoce haber sentido incertidumbre sobre su nivel de implicación y compromiso:

Cuando llegué, la antigua compañera me dijo: "aquello es horrible, te meten en una clase con un montón de niños y te dejan alli (se ríe mucho), di a todo que no, di a todo que no...". (Maestra1, Mujer, Colegio A).

Además, reconocen cierto temor hacia el contexto social y las situaciones de vulnerabilidad social del alumnado que asiste:

Hombre claro, al principio me asusté, claro (...).La primera vez me chocó la policía en la puerta (del colegio), me chocó bastante, no estaba acostumbrado a ver eso, y después las familias en bata, o en pijama, que llegan al cole, con sus babuchas (Maestro1, Hombre, Colegio B).

Esta sensación de incertidumbre es común en ambos centros, si bien una vez que se toma contacto con el proyecto, la disidencia es más fácil observarla en un caso que en otro. En la escuela A, de titularidad pública es fácilmente identificable, ya que en este centro es la administración educativa quien decide qué profesor o profesora irá a trabajar a través de un concurso público en el que se valoran sus méritos y no tanto sus deseos. Esto supone una amplia diversidad de situaciones por las que el profesorado llega a un centro y escasas repercusiones laborales el desvincularse del proyecto pedagógico emprendido. Esto provoca menos presión hacia el profesorado para expresar su adhesión o no al mismo y más dificultades para la configuración de equipos compactos:

Hemos venido por varias razones. Unas porque saben que venian al proyecto y se han informado, muchas porque les ha tocado. Muchas que les ha tocado, quieren permanecer y apuestan por él. Y además se integran en el 
proyecto, en fin. Entonces con algunas ha habido suerte y otras, menos suerte (Directora, Mujer, Colegio A).

En el centro concertado (Colegio B) es la dirección del colegio quien contrata al profesorado, por tanto, existen certezas compartidas sobre las obligaciones exigidas. Esto hace que la incorporación al proyecto de cambio se perciba como algo consensuado, con ideales armonizados, expresados en manifestaciones como "todos en el mismo barco" (Directora, Mujer, Colegio B).

Una vez que se ha tomado contacto con el proyecto y conforme avanza su desarrollo, el discurso del profesorado de ambos centros deja de aludir a cuestiones de índole individual en favor de cuestiones más grupales como el trabajo en equipo, la unidad, el compañerismo o diferentes solidaridades. El proyecto de cambio se empieza a valorar como algo que enriquece individualmente, les sostiene como equipo y compromete socialmente:

Ese apoyo que recibo es un alivio muy grande... Saber que vas a tener un problema pero vas a tener ayuda es una tranquilidad (Maestra2, Mujer, Colegio A)

Aqui hay un claustro de profesores muy comprometido con el entorno, el barrio, los niños, que no varían, que llevan eso pues 21 años, 30, muchos años y eso da estabilidad al centro (Maestra2, Mujer, Colegio B).

En ambos casos, esta mayor conciencia colectiva se produce a través de la internalización de una cultura compartida que se despliega a través de dos estrategias prioritarias: a) la formación continua del profesorado y b) la reflexión constante y compartida sobre su práctica. Con respecto a la formación del profesorado, en los dos contextos analizados ésta se concibe para el centro y no como una actividad o un beneficio meramente individual. Sin embargo, percibimos ciertas diferencias en el origen y el contenido de las solicitudes de formación. En el centro A, la demanda emerge de las necesidades detectadas por el equipo docente que idean estrategias, contenidos y acciones en paralelo a su actividad diaria:

Si tu pides un curso de formación, saben que le van a dedicar el mayor tiempo posible y que luego lo van a llevar a la práctica, que no es como esos cursos que a veces nos apuntamos... que después vas a las 4, 5 clases y que después no vas, y lo hacen personas por tener horas... (Directora, Mujer, Colegio A).

Pero en el colegio B, las acciones formativas surgen frecuentemente de la dirección, por lo que tiene una orientación más jerarquizada y, además, su contenido se vincula de forma explícita al ideario del centro, en este caso de carácter religioso:

Sí hay un perfil y una formación todos los años. Todos los años a principios de curso bay un encuentro que le llamamos Encuentro de Profesores Nuevos, y entonces a ese encuentro, es como un curso intensivo de fin de semana pues para impregnar a la gente que llega por primera vez y que conoce o no conoce el centro, pues del espiritu (nombre del fundador). Y luego pues también hay un seguimiento por parte de la titularidad, por parte del equipo directivo ¿eh? (Directora, Mujer, Colegio B).

Con relación a los procesos reflexivos, ambos centros se nutren del diálogo a través de instrumentos como las "asambleas" o las "reuniones de equipo" que llevan al profesorado a encontrarse de forma periódica (semanalmente por lo habitual). En el caso del colegio B, estas reuniones se desarrollan como parte de su compromiso con la institución concertada. En el colegio A, las reuniones se celebran ante la necesidad de fundamentar y deliberar entre todo el profesorado la toma de decisiones analizando y evaluando. De este modo, tienen la certeza de aprender con la experiencia, con el proceso de colectivizar tanto los logros como los fracasos. En ambos casos, la dimensión individual se diluye a favor de lo colectivo, atendiendo tanto a lo académico como a los valores educativos.

(...) si ha habido muchos cambios... es que yo ya no sé si ha habido cambios en general en el colegio o cambios en mi. Yo sí sé que he crecido mucho, que me he ilusionado mucho como profesional porque aqui lo que me he 
dado cuenta es que sí es posible otro tipo de organización, de gestión, de mirar los espacios, los tiempos, las agrupaciones ¿no? (Maestra3, Mujer, Colegio A)

Entrevistadora: ¿cuál crees que es la clave para que funcione el proyecto de Comunidad de Aprendizaje? Directora: el equipo, sí, este colegio sale adelante gracias al claustro de profesores que hay (Directora, Mujer, Colegio B).

Paralelo a este proceso de socialización colectiva, observamos altos niveles de certeza sobre el proyecto de cambio emprendido cuanto más estructurado está el proyecto. Así, el colegio B, a pesar de que el proyecto está en un nivel poco desarrollado, sigue un modelo muy planificado tanto desde fuera (por sus idearios intelectuales1), como a nivel interno (la estructura jerárquica de la organización a la que pertenece el centro), lo cual produce certezas que llevan al profesorado a confiar en sus virtudes:

Pero nos queda mucho tiempo para nosotros mostrar el colegio como $C d A$, lo mostramos como colegio que está iniciado en el proceso de comunidades de aprendizaje. Y no sé si se aprende más o menos, pero el clima, la convivencia, la interacción entre ellos sí que... que cambia (Maestra4, Mujer, Colegio B).

Por el contrario, en el colegio A, que ha seguido un modelo propio inspirado en la pedagogía experiencial y democrática, las certezas sobre la idoneidad del cambio y las estrategias que se siguen no resultan tan inmediatas. Ya que a pesar de tener una mayor trayectoria en el proyecto y apoyarse en la acción colectiva, muchos de estos cambios se ensayan y evalúan conforme se van desarrollando, por lo que la incertidumbre sobre los beneficios de los resultados es mayor.

Digo el primer año por momentos creíamos que... en algún momento dudamos que íbamos a conseguir cambios, pero en fin, acabó mal... más bien que mal el curso. Logramos conseguir y a partir de navidad cierto carisma con los niños y hacer un montón de cosas y actividades y entonces ya, un poquito cambió. (Maestro2, Hombre, Colegio A)

Las dificultades para desarrollar sus proyectos se manifiestan de forma diferente en cada contexto, aunque en ambos tiene que ver con la posibilidad de crear equipos. En la escuela A, tienen que ver con la falta de estabilidad de la plantilla, profesorado itinerante que impide avanzar de forma conjunta. En el caso del colegio B, donde la estabilidad es mucho mayor, las dificultades se orientan más hacia la coincidencia o no de intereses entre el profesorado y la estructura jerárquica:

(...) entonces se visitan estos centros, se traen personas de estos centros a formar a nuestro profesores en cursillos de dos dias, de tres días, y esto no se acaba sino que esto ya es una cadena: primero llega a unos pocos, después esto se imparte al total del profesorado, y entonces pues todo lo que se va descubriendo a lo mejor empieza por un grupito que se forma primero, pero la intención y la realidad es que luego esta formación llegue a todo el claustro (Maestra4, Mujer, Colegio B).

\subsection{La dimensión inter: diálogos con familias y entorno}

Las distintas estrategias, la finalidad y la valoración sobre las relaciones establecidas por los colegios con las familias y la comunidad permiten interpretar el discurso del profesorado desde la dimensión inter.

El objetivo del diálogo, según el profesorado de ambos centros, es mejorar la convivencia y conseguir escuelas de calidad. Las relaciones de apertura hacia el entorno se propician a través de actividades que no son espontáneas o anecdóticas, sino que vienen acompañadas de procesos planificados, diseñados y analizados dentro de la programación escolar, antes y después de su desarrollo. Sin embargo, como ha sucedido con anteriores aspectos, encontramos diferencias entre los dos colegios.

${ }^{1}$ Nos referimos al Grupo CREA y su desarrollo en el marco académico http:/ / comunidadesdeaprendizaje.net/ 
El centro A procura fomentar, por un lado, estrategias en las que las familias se hagan visibles tanto dentro de las aulas como en otros espacios de la escuela (teatro de madres, desayunos diarios, por ejemplo). Además, promueven la presencia del centro en el entorno inmediato, por ejemplo, a través de salidas del alumnado al barrio para la limpieza de calles o plazas, el trabajo con ONGs e instituciones o encuentros en diferentes acontecimientos de la ciudad y de relación con otros colegios. Su intención es difuminar las fronteras entre el centro escolar y el contexto social y familiar en el que se integra:

$Y$, sí, yo creo que los niños, el barrio creo que lo ha asimilado ya, el barrio está acostumbrado a ver a los niños preguntando, entrando a los lugares a preguntar. Yo creo que lo tienen asimilado y el verlos. (Maestra8, Mujer, Colegio A).

Dicha proyección hacia la comunidad posibilita una pluralidad de voces sobre expectativas diversas de profesorado y familias. Asimismo, da lugar a nuevas formas de expresarse y reconocerse más allá del déficit dentro y fuera del centro. Estas situaciones generan diálogos bidireccionales que tienen consecuencias en el discurso del profesorado. Las más evidentes pasan por destacar el reconocimiento que reciben de las familias, de agentes externos y administraciones públicas cuando expresan cómo ha cambiado la imagen que tienen del centro, pasando de ser problemático a percibirlo como un colegio innovador, alternativo y eficiente. Incluso reconocen que se sienten referente para instituciones como la universidad, con quienes establecen redes de colaboración e investigación de forma constante.

(...) nos invitan colegios de otras zonas que llevan amigos míos y dicen: ¿tu colegio es este de (nombre del centro) y tal? Es que json niños fabulosos! y les digo, claro que son fabulosos, o sea, están en un ambiente difícil pero no dejan de ser niños y niñas con sus características y por supuesto que son fabulosos como los que tú tienes en tu centro (Maestro9, Hombre, Colegio A).

El diálogo y proyección hacia el exterior en el Colegio B también está presente con el proyecto CdA. Sin embargo, se encuentra mediado por dos factores: (1) el nivel de estructuración y diseño del proyecto, lo que les ha llevado a realizar estrategias de apertura muy protocolizadas, ceñidas al espacio del aula y vinculadas fundamentalmente al desempeño académico del alumnado; y (2) la acentuada disposición por respetar el ideario religioso del centro dicta unos valores que se proyectan inalterables y suponen el umbral sobre el cual las familias deben decidir y aceptar si desean matricular o no a sus hijos y, por tanto, a participar y aceptar lo que el centro disponga:

No, por eso te digo, que con lo que es el alumnado inmigrante no tenemos problema, ellos respetan ¿sabes?, cuando vamos a la iglesia o cuando vamos a la capilla, ellos asisten, respetan, (...) ellos desde un principio cuando vienen al colegio se les dice que aqui esto es un colegio católico. (...). Lo que si tenemos más problemas es con el alumnado gitano, (..) y claro, ellos saben, no se les engaña. Entonces pues bueno, pero sí es cierto que cuando bay actividades, por ejemplo, el tema de la Coronación a María, abi pues siempre hay pues el típico niño pues que rechaza (...). Pero es que es lo que aceptan desde el principio, desde que se matriculan aqui (Maestra7, Mujer, Colegio B).

Estos factores en el centro B, aunque limitan, no impiden que se esté produciendo una mayor relación con las familias y la comunidad, y que se destaque el reconocimiento sobre su trabajo al igual que en el centro A.

No obstante, el uso que se hace de dicho reconocimiento es distinto. El colegio B lo utiliza no solo para dar mayor legitimidad a sus prácticas, sino para darle mayor prestigio y estatus frente a otros centros y así aumentar la matrícula de alumnado. Mientras, el profesorado del Centro A, asume estos reconocimientos como oportunidades para consolidar el proyecto a través de peticiones concretas como, por ejemplo, estabilizar la plantilla u obtener más recursos de la administración pública. 


\section{Discusión}

Este trabajo enriquece el campo de estudio sobre profesorado como activista, líderes comprometidos y colaborativos que desarrollan formas de conocimiento y acción para la mejora de las escuelas desde la reflexión y la acción (Aguado-Odina, Mata-Benito y Gil-Jaurena, 2017).

La lectura de los resultados señala, en primer lugar, que el trabajo en equipo es el eje del cambio para la equidad. Los contextos de dificultad y desigualdad social impulsan la generación de equipos, al unirse para superar las dificultades, pero también se presentan como elementos de dificultad y abandono del proyecto en algunos casos. Se observa cómo la jerarquía organizativa del Centro B, de titularidad concertada, ha generado un mayor nivel de adhesión al proyecto que la del centro A público, favorecido por mecanismos como la contratación directa y su presencia más estable en la escuela.

En segundo lugar, y en referencia al proceso de acomodo, destacan que conforme avanza el proceso de cambio las certezas del profesorado sobre las mejoras emprendidas aumentan, especialmente si el proyecto está sistematizado y pautado.

En tercer lugar, en ambos casos y a pesar de las diferencias, los proyectos se consolidan con estrategias como la formación colectiva y la reflexión conjunta. El profesorado internaliza valores y principios comunes que generan una cultura profesional compartida (Moliner et al., 2016).

Estos tres elementos no difieren de lo hallado en otros trabajos sobre cambio escolar en contextos de desigualdad (Wrigle et al., 2012), en los que se subraya la importancia del liderazgo pedagógico distribuido y la cultura de la colaboración para los procesos mejora. Dichos elementos generan para los actores implicados construcciones individuales y colectivas sobre significados que tiene el cambio, catalizando la innovación escolar con proyectos alternativos (Philpott y Oates, 2017). Pero, además, estos procesos de cambio sirven para generar certezas en el profesorado, sobre sus propias prácticas, individuales y colectivas, que las orientan a una mayor apertura hacia el trabajo en equipo. Como señalan Van der Heijden et al. (2018), al construir la visión de una escuela para el futuro, los docentes se sienten apegados a la organización y motivados para combinar sus metas con las de la organización.

En cuarto lugar, también nuestros resultados coinciden con investigaciones que señalan que las iniciativas hacia la mejora, el cambio o la innovación escolar en contextos de desigualdad y diversidad cultural, promueven de forma reiterada actividades que permiten crear y fortalecer el vínculo con las familias y otros actores del entorno (organizaciones, asociaciones o universidad) (Wrigle et al., 2012; Sleeter et al., 2016). Sin embargo, en nuestro estudio observamos, por un lado, cómo la titularidad del centro y la percepción frente al interlocutor inciden en los modos en los que esta relación se lleva a cabo. En el caso A, el diálogo bidireccional entre el centro y las familias promueve la disolución de fronteras y la creación de vínculos entre ámbitos y actores (aula, centro, comunidad, profesorado, alumnado, familias, otras entidades) a un mismo nivel. En el otro caso, al priorizarse el ideario religioso del centro sobre el de las familias, se dificulta el encuentro multicultural (González et al., 2005). Por otro lado, no hemos encontrado suficientes datos que nos permitan mostrar ejemplos de construcción de un curriculum que capitalice las lenguas o procesos identitarios alternativos o enfrente de manera explícita los temas de equidad y poder, como se señalaba en la tercera y cuarta dimensión de Sleeter et al. (2016) para los proyectos de equidad y justicia social. Las actividades con las familias suponen una apertura del colegio a estos nuevos actores, pero aún sigue presente -más en el colegio privado-concertado que en el público- una perspectiva excesivamente escolar-céntrica en lo referente al currículum escolar.

A pesar de ello coincidimos con la literatura que señala que estos procesos dialógicos posibilitan la superación de prejuicios, combaten la dimensión cultural del déficit y favorecen las concepciones sobre la diversidad en el profesorado (Hakisoteriou y Angelides, 2017; Subero et al., 2015; Sleeter et al., 2016). Es decir, posibilitan el reconocimiento y la pluralidad de identidades del alumnado y sus familias, no percibiéndolos exclusivamente como "pobres" o "excluidos", condenados al fracaso escolar o al desempleo. 
En quinto y último lugar, la percepción del reconocimiento aparece como elemento destacable en los proyectos de cambio escolar para la equidad y la justicia social en ambos casos. El profesorado destaca que sus prácticas son legitimadas por parte de la comunidad y objeto de un reconocimiento público por parte de la administración y población que acude al centro. Dicho reconocimiento no sirve para atraer alumnado a los centros educativos de otro perfil, pero sí como una oportunidad para contextualizar el fracaso en el marco de la desigualdad estructural más allá de señalarlos como responsables de su propio destino. De este modo, los proyectos de cambio escolar orientados a la equidad y la justicia social adoptan un "valor añadido" a los centros, es decir, tienen un valor superior al previsible teniendo en cuenta los contextos y el punto de partida de fracaso y conflicto (SilasCasillas y Perales-Franco, 2014).

\section{Conclusiones}

Este trabajo ha permitido ilustrar y analizar en profundidad procesos de cambio escolar que revelan dinámicas calificadas por Wrigley et al., (2012) como "pedagogía de la iluminación". Escuelas que se orientan a la mejora y en las que el profesorado es eje central para ayudar a los jóvenes individual y colectivamente a constituir un nuevo imaginario social, un futuro mejor y un mayor reconocimiento de la pluralidad de identidades sociales. El compromiso social, ético y político del profesorado adquiere una relevancia esencial y sus prácticas, con las certezas e incertidumbres que se generan, resultan cruciales para la transformación a favor de la equidad.

Las limitaciones de este estudio se superarían aumentando el número de casos y la comparación con otros contextos. Además, realizar trabajos de campo durante más tiempo triangulando estrategias de investigación como las observaciones o el análisis de documentos permitiría comprender la proyección de las estrategias particulares adoptadas en cada una de las fases de los proyectos emprendidos, así como profundizar en el desarrollo curricular en las aulas de forma más pormenorizada. Este tipo de trabajos son relevantes para comprender en mayor profundidad la cultura del cambio escolar orientada a la equidad más allá de ingenierías sociales, estándares y modelos protocolizados ajenos a contextos locales que implican a sujetos concretos, procesos y objetivos particulares y comunes de un futuro deseable.

\section{Referencias bibliográficas}

Aguado-Odina, T., Mata-Benito, T., \& Gil-Jaurena, I. (2017). Mobilizing intercultural education for equity and social justice. Time to react against the intolerable: A proposal from Spain. Intercultural Education, 28(2), 1-16. doi: 10.1080/14675986.2017.1333874

Ayers, W., Quinn, T., \& Stovall, D. (Eds.) (2009). Handbook of social justice in education. Nueva York: Routledge

Creswell, J. W. (2007). Qualitative inquiry \& research design: Choosing among five approaches. Thousand Oaks, CA: Sage Publications, Inc

Essomba, M.A. (2006). Liderar escuelas interculturales e inclusivas. Barcelona: Graó.

Flecha, R., \& Soler, M. (2013). Turning difficulties into possibilities: engaging Roma families and students in school through dialogic learning. Cambridge Journal of Education, 43(4): 451-465. doi.org/10.1080/0305764X.2013.819068

García, R., Mircea, T,. \& Duque, E. (2010). Socio-Cultural Transformation and the Promotion of Learning. Revista de Psicodidáctica, 15(2), 207-222.

Gilad-Hai, S., \& Somech, A. (2016). “The day after”: The organizational consequences of innovation implementation in experimental schools. Journal of Educational Administration, 54(1), 19-40. doi:0.1108/JEA-07-2014-0084.

González, N., Moll, L., \& Amanti, C. (2005). Funds of knowledge. Theorizing practices in households, communities, and classrooms. Mahwah, NJ: Lawrence Erlbaum Associates. 
Hajisoteriou, C., Karousiou, C., \& Angelides, P. (2017). Successful components of school improvement in culturally diverse schools. School Effectiveness and School Improvement, 5, 1-22. doi: 10.1080/09243453.2017.1385490

Hajisoteriou, C., \& Angelides, P. (2017). Collaborative art-making for reducing marginalisation and promoting intercultural education and inclusion. International Journal of Inclusive Education, 21(4), 361-365. doi: 10.1080/13603116.2016.1197321.

Lupton, R. (2005). Social justice and school improvement: improving the quality of schooling in the poorest neighbourhoods. British Educational Research Journal, 31(5), 589-604. doi: 10.1080/01411920500240759

Moliner, O., Sales, A., \& Escobedo, P. (2016). Posibilidades y limitaciones de generar cultura de centro desde las prácticas compartidas de aula: el caso de la educación intercultural inclusiva. Revista sobre educación, 30, 51-70. doi.org/10.15581/004.30.51-70

Philpott, C., \& Oates, C. (2017). Professional learning communities as drivers of educational change: The case of learning rounds. Journal Education Change 18, 209-234 doi.org/10.1007/s10833-0169278-4

Ragin, C. (1987). The comparative method. Moving beyond qualitative and quantitative strategies. Berkeley: University of California Press.

Sammons, P. (1999). School effectiveness: coming of age in the 21st century. Abingdon: Swets \& Zeitlinger.

Silas-Casillas, J.C., \& Perales-Franco, C. (2014). Making a difference in poor communities: relations among actors in Mexican schools. International Journal of Qualitative Studies in Education, 27(8), 9921019. doi.org/10.1080/09518398.2014.924639

Sleeter C., Montecinos, C., y Jiménez, F. (2016). Preparing teachers for social justice in the context of education policies that deepen class segregation in schools. The case of Chile. En J. Lampert y B. Burnett (Eds.). Teacher education for high poverty schools (pp. 171-191). Cham: Springer.

Subero, D., Vila, I., \& Esteban-Guitart, M. (2015). Some Contemporary Forms of the Funds of Knowledge Approach. Developing Culturally Responsive Pedagogy for Social Justice. International Journal of Educational Psychology, 4(1), 33-53. doi.org/10.4471/ijep.2015.02

Van der Heijden, H.R.M.A., Beijaard, D., Geldens, J.J.M., \& Popeijus, H.L. (2018). Understanding teachers as change agents: An investigation of primary school teachers' selfperception. Journal of Educational Change, 19(3), 347-373. Doi:10.1007/s10833-018-9320-9

Wrigley, T. (2011). Paradigms of school change. Management in Education, 25(2), 62-66.

Wrigley, T., Lingard, B., \& Thomson, T. (2012). Pedagogies of transformation: keeping hope alive in troubled times. Critical Studies in Education, 53(1), 95-108. doi.org/10.1080/17508487.2011.637570

\section{Para citar este artículo}

García-Cano Torrico, M., y Márquez Lepe, E. (2019). Estrategias de docentes en centros educativos que se transforman a favor de la equidad. Revista Fuentes, 21(2), 221-227. [Fecha de consulta: dd/mm/aa]. doi: 10.12795/revistafuentes.2019.v21.i2.06. 